\title{
Le système eau-pyroglutamate de zinc : déshydratation et réhydratation des composés de coordination
}

\author{
P. Espeau, A.M. Loireau-Lozac'h, H. Allouchi ${ }^{1}$ et R. Céolin
}

Laboratoire de Chimie Physique, Université Paris V, Faculté des Sciences Pharmaceutiques et Biologiques, 4 avenue de l'Observatoire, 75006 Paris, France

${ }^{1}$ Laboratoire de Chimie Physique, Faculté de Pharmacie, 31 avenue Monge, 37200 Tours, France

\begin{abstract}
The relative stabilities of the (S-S) and (R-R, S-S) diaquabis(pyroglutamato)zinc(II) phases, both derived from density and solubility data, and thermal behaviour under pressure, are discussed. These phases dehydrate into amorphous and crystalline anhydrous phases upon heating, which rehydrate at room temperature under saturating water vapour through mechanisms that depend on crystallinity and presumably on diastereo-isomerism.
\end{abstract}

\section{1- INTRODUCTION}

L'oligo-élément zinc peut être vectorisé dans un but thérapeutique sous forme de composés de coordination. L'un d'eux, obtenu par réaction entre l'oxyde de zinc et l'acide (S)-pyroglutamique en solution aqueuse, est le diastéréoénantiomère (S-S) du diaquabis (pyroglutamato)zinc(II) (composé I). Sa structure cristalline (1) montre que l'atome de zinc est au centre d'un tétraèdre d'atomes d'oxygène dont deux proviennent de molécules d'eau (Fig. 1).

La réaction entre $\mathrm{ZnO}$ et l'acide optiquement inactif conduit au racémate (R-R, S-S) du diaquabis(pyroglutamato)zinc(II) (composé II) dont la structure cristalline (2) montre le même environnement tétraédrique de l'atome de zinc.

La caractérisation des composés I et II a été entreprise dans un but de préformulation pharmacotechnique.

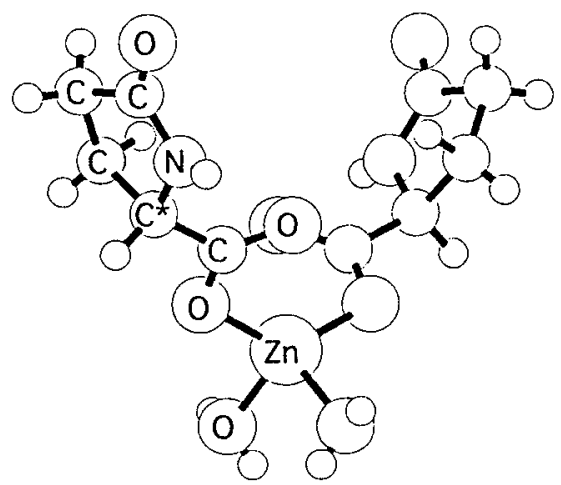

Figure 1 : diastéréoénantiomère (S-S) du diaquabis(pyroglutamato)zinc(II)

\section{CARACTERISATION STRUCTURALE ET THERMODYNAMIQUE DE (I) ET (II)}

Les profils de diffraction des rayons X des composés I et II, obtenus après évaporation de leurs solutions aqueuses, sont identiques aux profils calculés à partir des structures cristallines. 
L'échauffement de I et II $\left(5 \mathrm{~K} \cdot \mathrm{min}^{-1}\right)$ montre que leur déshydratation débute sensiblement vers $400 \mathrm{~K}$ (Fig. 2). Les enthalpies de déshydratation, très voisines de $120 \mathrm{~kJ} \mathrm{~mol}^{-1}$ dans les deux cas, correspondent aux valeurs attendues pour la perte, vérifiée par ATG, des deux molécules d'eau de coordination.

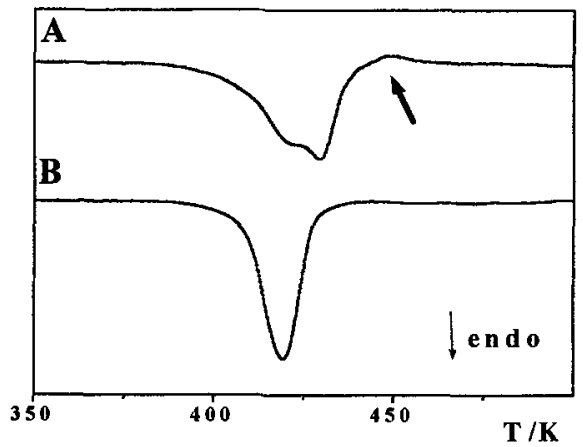

Figure 2 : Signaux DSC de la déshydratation de I (A) et $\mathbf{I}$ (B)

La phase obtenue après déshydratation de $I$ est une phase non cristalline (composé I'nc) qui recristallise lorsque l'échauffement se poursuit. Ce processus se traduit par un effet exothermique (flèche sur la courbe A, Fig. 2). L'enthalpie associée à l'obtention de la phase anhydre cristallisée (composé I'cr) vaut environ $-11 \mathrm{~kJ} \mathrm{~mol}^{-1}$. La phase obtenue après déshydratation de II est une phase non cristalline (composé II'nc) qui le reste jusqu'à sa dégradation thermique. La dissolution des phases anhydres I'nc, I'cr et II'nc puis l'évaporation des solutions à $298 \mathrm{~K}$ conduisent aux phases initiales hydratées I et II cristallisées.

Les solubilités des composés I et II ont été mesurées par gravimétrie (Fig. 3).

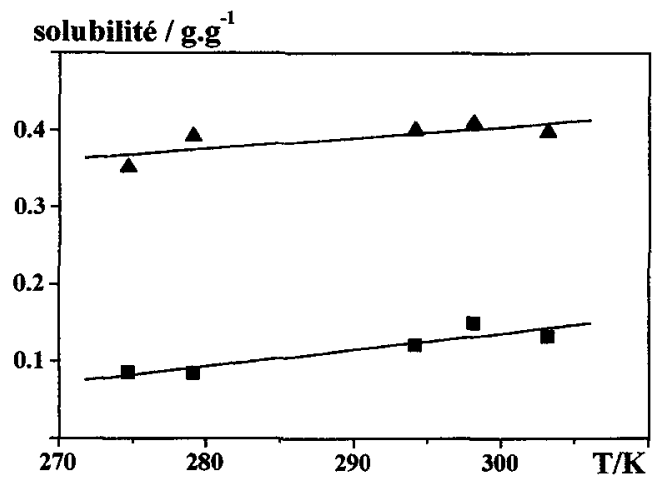

Figure 3 : Courbes de solubilité (en grammes de composé par gramme d'eau) en fonction de la température (W : composé I, A : composé $\mathbf{I})$ 


\section{STABILITES RELATIVES DES COMPOSES I ET II}

D'après les études cristallographiques $(1,2)$, le composé I (diastéréoénantiomère (S-S)) est plus compact que le composé II (racémate (R-R,S-S)) : V/Z(I) $=342$ et V/Z(II) $=347 \AA^{3} /$ molécule.

Ces données s'accordent avec la règle selon laquelle plus grande est la compacité plus grande est la stabilité. Toutefois, de nombreuses exceptions (3) appellent la recherche d'autres critères.

La règle selon laquelle plus le point de fusion est élevé plus grande est la stabilité ne peut être utilisée dans le cas des composés I et II qui se déshydratent avant de fondre. C'est pourquoi leurs solubilités S ont été mesurées. La Fig. 3 montre que le composé II est environ quatre fois plus soluble que le composé $I$ et que les courbes $S=f(T)$ sont sensiblement parallèles dans l'intervalle de température exploré (273-303 K). Le point de fusion de I serait alors plus élevé que celui de II. Par suite, I serait plus stable que II au sens de Jacques, Collet et Wilen (3).

Cette conclusion semble s'accorder avec les résultats obtenus en "DSC sous pression" (capsules étanches en acier inox). Les températures (début et maximum) de l'endotherme enregistré à l'échauffement du composé I sont plus élevées que celles trouvées avec le composé II (I : 442 et $448 \mathrm{~K}$, II : 408 et $425 \mathrm{~K}$ ). Toutefois, seule l'étude complète des systèmes binaires eau-I' et eau-II' pourrait permettre de déterminer si les fusions ainsi observées sont congruentes ou non.

\section{REHYDRATATION DES COMPOSES ANHYDRES I'nc, I'cr ET II'nc}

Trois techniques ont été utilisées pour observer, à température ambiante et sous tension saturante de vapeur d'eau, la réhydratation des phases anhydres, cristallines ou non : calorimétrie, gravimétrie et diffraction des rayons $\mathrm{X}$.

En calorimétrie isotherme, les exothermes pour I'nc et I'cr ont des profils semblables (Fig. 4A) bien que celui de I'nc corresponde à un dégagement de chaleur $\left(120 \mathrm{~kJ} \cdot \mathrm{mol}^{-1}\right)$ supérieur à celui de I'cr $\left(110 \mathrm{~kJ} \cdot \mathrm{mol}^{-1}\right)$. Cette inégalité paraît être associée (selon le premier principe de la thermodynamique) à la transformation $I^{\prime} n c \rightarrow I ' c r$. Toutefois, le suivi isotherme par diffraction des rayons $\mathrm{X}$ (Fig. 4B) ne montre pas la présence transitoire de I'cr mais seulement celle des pics de I après un temps de latence supérieur à celui observé en calorimétrie. Autrement dit, I'ne s'hydrate en formant I (comme le montre aussi l'ATG) sans passer par l'étape I'cr. On note de plus que I'nc se transforme en I plus précocement mais moins rapidement que ne le fait I'cr (Fig. 4B).
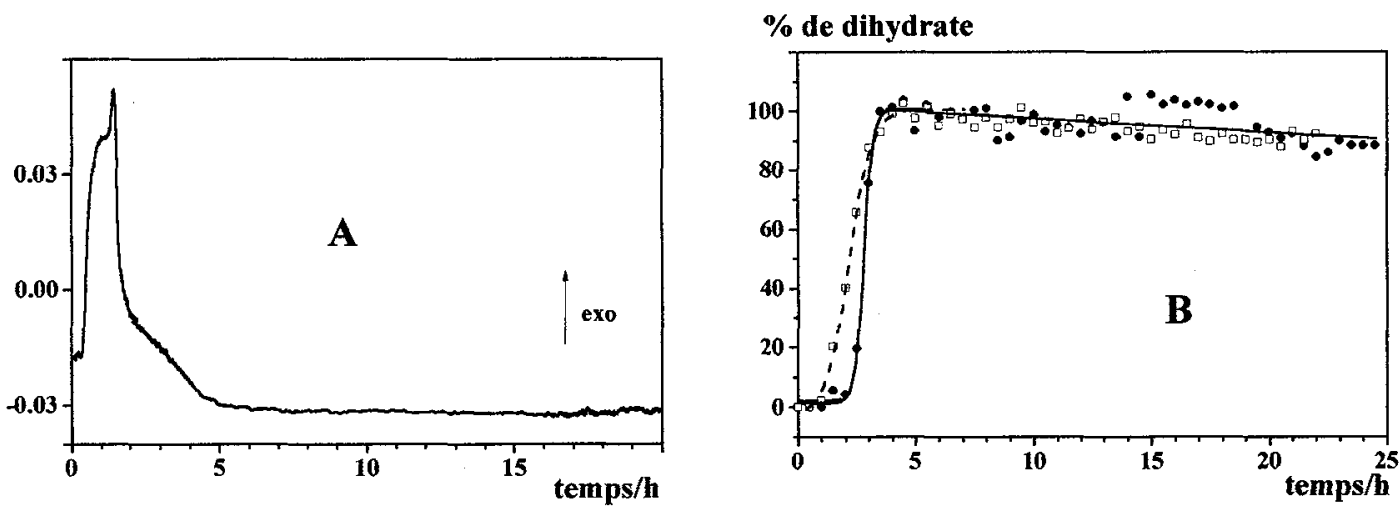

Figure 4 : Réhydratation isotherme de I'nc et I'cr en fonction du temps. A : exothermes observés en DSC. B : suivi par RX de la formation des dihydrates à partir de I'nc $(\square)$ et I'cr $(\bullet)$. Pourcentages établis par mesure de la somme des intensités relatives de pics de Bragg en fonction du temps 
L'exotherme associé à l'hydratation de II'nc (Fig. 5A) est différent de celui enregistré avec I'nc. Il semble en effet composé de deux parties séparées par un effet endothermique dont la fin coïncide avec l'apparition soudaine de II, comme le montre la diffraction des rayons X (Fig. 5B). Quoi qu'il en soit, l'intégration de l'ensemble des effets thermiques montre que la chaleur dégagée lors de l'hydratation est voisine de $120 \mathrm{~kJ} \cdot \mathrm{mol}^{-1}$.
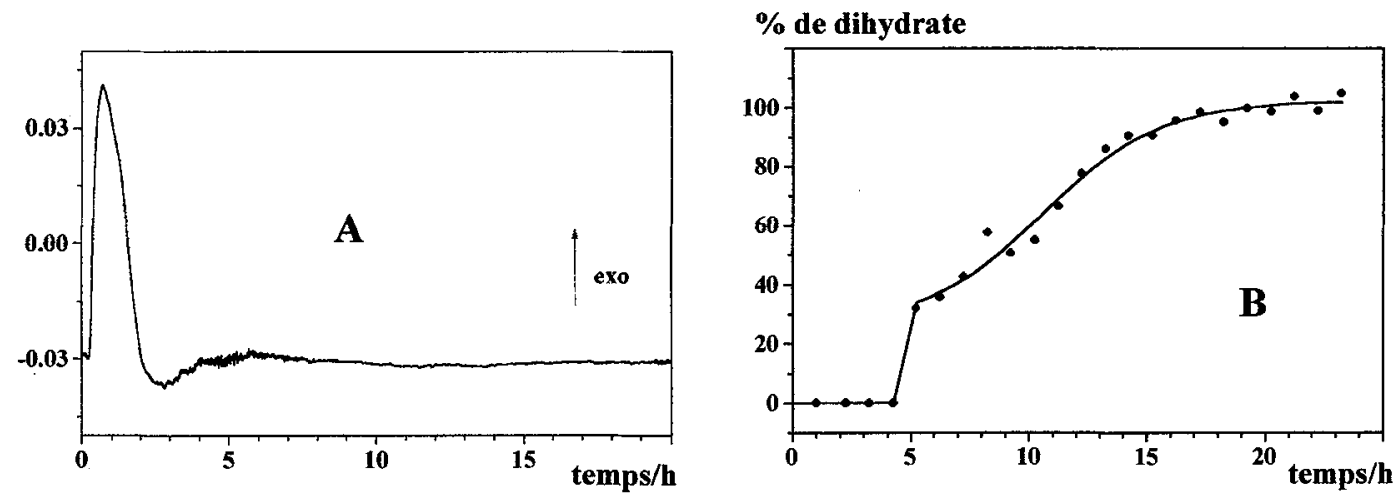

Figure 5 : Réhydratation isotherme de Il'nc en fonction du temps. A : exothermes observés en DSC. B : suivi par RX de la formation du dihydrate

Ces résultats indiquent que l'hydratation isotherme de I'nc, I'cr et I'nc relève de mécanismes qui diffèrent d'un composé à l'autre non seulement à cause de la cristallinité mais aussi, peut-être, de la diastéréoisomérie. Ces différences impliquent que les environnements du zinc dans I'nc, I'cr et II'nc peuvent être différents les uns des autres. Afin de vérifier cette hypothèse, des expériences EXAFS visant à déterminer l'environnement du zinc dans ces composés ont été entreprises.

\section{Références}

[1] N. Rodier, R. Céolin, M. Plat, H. Zumbihl, Acta Cryst. C46 (1990) 324-326.

[2] A.M. Loireau-Lozac'h, R. Céolin, X. Solans, M. Font-Bardia, Acta Cryst. C54 (1998) 1571-1573.

[3] J. Jacques, A.Collet, S.H. Wilen, Enantiomers, Racemates and Resolutions (Wiley-intersciences publication, 1981). 